\title{
Robot Behavioral Mapping: A Representation that Consolidates the Human-robot Coexistence
}

\author{
Ioannis Kostavelis* \\ Department of Production and Management Engineering, Democritus University of Thrace, Greece
}

Submission: August 17, 2017; Published: September 07, 2017

*Corresponding author: Ioannis Kostavelis, Production and Management Engineering Department, Democritus University of Thrace, Vas Sophias 12, Xanthi, 671-32 Greece, Email: gkostave@pme.duth.gr

\begin{abstract}
The paper at hand aims to introduce for the first time the concept of robot behavioral mapping, which is an amalgamated environment and human representation suitable for long term navigation planning, task scheduling and decision making. The robot perception mechanisms comprised the cornerstone for metric and semantic mapping, which gave thrust to the development of robots capable of apprehending the operational environment in a human compatible manner. Yet, safety is a mandatory ingredient for the human-robot cohabitation and, thus, social mapping became a momentous element in the robotics community, where the human presence is modeled along with the environment. The behavioral mapping constitutes the evolution of the aforementioned technologies in the near future, aiming to consolidate an additional dimension to the domain of robotics perception in accordance which, robot companions should shape behavioral conformation for living with people.
\end{abstract}

Keywords: Behavioral mapping; Robot-centric mapping; Human-centric mapping

\section{Introduction}

The interest of the film industry in making movies such as the Bicentennial Man [1999], Robot \& Frank [2012] and many others reveals the broad acceptance, as well as the comprehension of people for domestic and service robots in near future communities. Such movies disclose people's vision about artificial agents to be released to the market within the upcoming years.

However, according to the Gartner's Hype Cycle for Emerging Technologies [1], smart robots are still at the middle of the innovation trigger and it is anticipated a 5 to 10 years time interval to reach the plateau of productivity. One of the main parameters dragging this technology back is the fact the smart robotic agents shall have to simultaneously confront not only humans, but their environments, their manners and their social structure as well. This in turn generates a complex grid of requirements that demands for a multi-faceted treatment. Therefore, motivation beneath this paper is to outline the basic objectives that robot behavioral mapping should retain in order to close the loop of the robot-human perception and modeling by exploiting the recent advancements in the fields of robot vision and artificial intelligence. A way to tackle this problem is to create robots that obey to the following three principals, which constitute the outline of this conceptual work: a. The robots should be able to understand, interpret and represent their environment in a human compatible manner

b. The robots should be able to apprehend human occurrence and activities, so as to act accordingly in human inhabited environments.

c. The robots should retain a behavioral model acting as mediator that facilitates seamless cooperation among the two aforementioned principals.

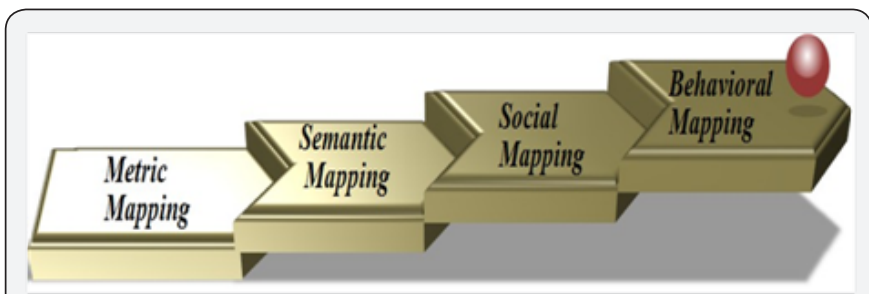

Figure 1: A hierarchical representation of the existing mapping methods and the association with the robot behavioral mapping.

The ultimate objective in this work is to exhibit the way robot behavioral mapping will receive major attention from the robotics community the upcoming years by highlighting its prominent characteristics. Therefore, this work organizes the existing mapping techniques as robot-centric ones that include 
metric and semantic mapping strategies and human-centric ones that consists of social mapping and the newly introduced here behavioral mapping methods. The Figure 1 provides a schematic representation of the relationship among the existing mapping strategies with the behavioral mapping one.

\section{Metric mapping}

This type of mapping comprised the cornerstone of the robot perception as it provides a geometric representation of the explored environment that endows the robots with awareness about the spatial layout of their working area [2]. For example the robot's with metric mapping capacities are able to understand that the explored environment is a surface with $\mathrm{Sm}^{2}$ dimensions and some of it is occupied by obstacles while the rest of it is empty space. The well defined Simultaneous Localization and Mapping (SLAM) according to which a mobile robot placed at any unknown location in an unexplored area incrementally builds a consistent map of the environment while simultaneously determines its location within this map $[3,4]$ allowed the robots to enter the industrial and domestic environments and work with humans, yet with limited capabilities.

\section{Semantic mapping}

These capabilities were increased since the robots obtained the capacity to apprehend their environment in a humancompatible manner. Semantic mapping provided the means to achieve this by associating human concepts, such as types of objects and places, with their spatial arrangement extracted from the environment's metric map [5]. Semantic mapping offers a qualitative description of the robot's surroundings aiming to augment the navigation capabilities and the taskplanning relying on the capacity of the artificial agents to accurately recall the learned spatial memories. The robots with semantic mapping capacities are able to understand the explored area with surface of $\mathrm{S} \mathrm{m}^{2}$ corresponds to a "corridor" and the occupied cells are obstacles that correspond to objects such as "chairs" and "tables".

\section{Social mapping}

When the semantic map is augmented with human modeled actions it becomes the social aware mapping, where the robot can apprehend high level concepts that relate the explored environment with human presence [6]. The primary objective of the social mapping is to regulate the robot's navigation capabilities by constraining the operational environment in accordance with the human presence. When, this attribute is correlated with human actions in specific geometrical regions then, rule based navigational policies can be inferred by the robot's in order operate with comfort [7], naturalness and sociability [6]. When a robot is endowed with social mapping capabilities, it becomes able to apprehend that the explored area with surface of $\mathrm{S} \mathrm{m}^{2}$ corresponds to a "corridor" with "chairs and tables" and that "humans typically walk in a corridor".

\section{Behavioral mapping}

The behavioral part of robot mapping emerges the association of the agent's attitude within the explored environment considering the semantic and social characteristics of it. In its simple form, behavioral mapping comprises the monitoring of the human's presence in specific places during time and assigns to the respective spatial region a time-evolving occupancy label. Graphically speaking this map would be a 3D volumetric representation that corresponds to the cells' occupancy, yet with a specific time step. Such a representation corresponds to the levels of humans' congestion within particular regions of the metric map for a pre-defined time window e.g. one day. In a more complex illustration, a behavioral map represents the human actions as a $3 \mathrm{D}$ volumetric representation where each voxel receives specific label related to a human action. Considering again the above mentioned example, a robot endowed with a behavioral map would be able to apprehend that the area with surface of $\mathrm{S} \mathrm{m}^{2}$ corresponds to a "corridor" with "chairs and tables", that "humans typically walk in a corridor" and that a "corridor is highly congested during morning and afternoon". Figure 2 illustrates a conceptual representation of the behavioral map which should be positioned on top of the existing robot and human-centric maps.

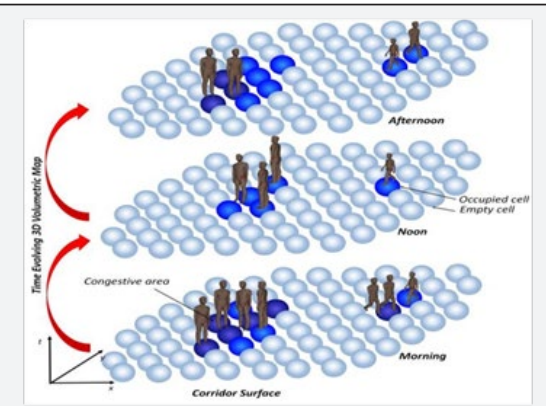

Figure 2: A conceptual representation of a time evolving robot behavioral map that considers the human presence during different time slots in the day.

\section{Added Value of a Behavioral Map}

Similarly to the way a metric map is utilized for robot navigation, a behavioral map can be used for the robot's higher level functionalities targeting on cohabitation with humans yet respecting the their comfort and retain naturalness during their coexistence increasing thus safety, autonomy and dependability. Robot behavioral conformation stands for the agent's capacity to interpret its surroundings considering the spatial and semantic attributes of the objects in relation to the human's presence and actions and to regulate its operational parameters respectively facilitating ambient and safe human robot cohabitation. Specifically, behavioral maps 


\section{Robotics \& Automation Engineering Journal}

are information reach representation that can be used from artificial agents:

a. To adjust robot's operational parameters such as velocity values and manipulation intervention levels especially when agent transits among places with variation in the human activity

b. For long term robot path planning, by considering timedepended specific trajectories avoiding congestive areas in rush hours maximizing safety when operating in human populated environments.

c. For robot decision making considering the scheduling of appropriate tasks given a time window. Specifically, the 3D volumetric representation of human occupancy comprises a convenient representation for various decision making algorithms constraining the environment with a mapping of human occupancy, considering the available robotic skills.

\section{Conclusion}

In this introductory paper the concept of the robot behavioral mapping as the future step in the field of robot perception has been presented. The necessity of the behavioral mapping for future service robots has been justified, while at the same time dependence and relation to its antecedents has been explained. Moreover, the added value of such a map has been outlined providing an indicative usefulness for the robot behavioral mapping.

\section{References}

1. Fenn J, LeHong H (2011) Hype cycle for emerging technologies, Gartner

2. Thrun S, Burgard W, Fox D (2005) Probabilistic robotics. MIT press Cambridge, London, UK.

3. Durrant-Whyte H, Bailey $\mathrm{T}$ (2006) Simultaneous localization and mapping: part I. IEEE Robotics \& Automation Magazine 13(2): 99-110.

4. Bailey T, Durrant-Whyte H (2006) Simultaneous localization and mapping (slam): Part II. IEEE Robotics \& Automation Magazine 13(2): 108-117.

5. Kostavelis I, Gasteratos A (2015) Semantic mapping for mobile robotics tasks. Robotics and Autonomous Systems 66(C): 86-103.

6. Charalampous K, Kostavelis I, Gasteratos A (2016) Robot navigation in large-scale social maps. Expert Systems with Applications 66(C): 261273.

7. Kruse T, Pandey AK, Alami R, Kirsch A (2013) Human-aware robot navigation: A survey. Robotics and Autonomous Systems 61(12): 17261743

\section{Your next submission with Juniper Publishers will reach you the below assets}

- Quality Editorial service

- Swift Peer Review

- Reprints availability

- E-prints Service

- Manuscript Podcast for convenient understanding

- Global attainment for your research

- Manuscript accessibility in different formats

(Pdf, E-pub, Full Text, Audio)

- Unceasing customer service

Track the below URL for one-step submission https://juniperpublishers.com/online-submission.php 